www. revistadyo.com

\title{
Análisis de la contribución científica Latinoamericana en la temática de los vehículos eléctricos
} Willmer Guevara Ramírez, Rosa-María Río-Belver, Itziar Martinez de Alegría,
Cristian Morales Letzkus

https://doi.org/10.37610/dyo.v0i75.610
Recibido: 12 de Enero de 2021

Aceptado: 5 de Abril de 2021

\section{Resumen}

Este trabajo tiene como objetivo analizar mediante un análisis bibliométrico la contribución científica latinoamericana en la temática de los vehículos eléctricos, utilizando las investigaciones indexadas en la Web of Science (WOS) en el periodo 2001-2019. Los resultados muestran poca contribución de los países latinoamericanos, sus organizaciones y autores, además de un bajo enfoque hacia las investigaciones sobre las baterías de litio. Lo que permite concluir que las organizaciones y agencias de financiamiento científico de la región deberían potenciar las investigaciones en la temática, favoreciendo los proyectos que proponga amplias redes de colaboración que permita una rápida transferencia de conocimiento.

\section{Palabras clave}

Vehículos eléctricos, Baterías de litio, Latinoamérica, Índice de actividad, Producción científica.

\section{Introducción}

Los vehículos eléctricos (en lo adelante "EVs", por sus siglas en inglés) surgieron como una contribución importante a dos problemáticas que ocupan la agenda de muchos países y organizaciones mundiales. En primer lugar, se encuentra el cambio climático y su impacto sobre los ecosistemas (Walther et al., 2002; Thomas et al., 2004). Entre sus principales causas están las emisiones de dióxido de carbono (CO2) asociadas a la quema de combustibles fósiles por parte de la industria y los medios de transporte (Fargione \footnotetext{
Willmer Guevara Ramírez *

iD https://orcid.org/0000-0001-9210-0786

Rosa-María Río-Belver *

rosamaria.rio@ehu.eus

iD https://orcid.org/0000-0002-4244-9098

Itziar Martinez de Alegría **

itziar.martinezdealegria@ehu.eus

iD https://orcid.org/0000-0003-0731-3010

Cristian Morales Letzkus ***

cmorales@ucn.cl

iD https://orcid.org/0000-0003-1414-8906

* University of the Basque Country (UPV/EHU) Faculty of

Engineering in Vitoria-Gasteiz Industrial Organization and

Management Engineering Department C/ Nieves Cano 12,

01007 Vitoria, Spain

** University of the Basque Country (UPV/EHU) Faculty of

Engineering in Vitoria-Gasteiz Industrial Organization and

Management Engineering Department Pl Ingeniero Torres

Quevedo 1, 48113 Bilbao, Spain

*** Escuela de Ciencias Empresariales, Universidad Católica

del Norte, Larrondo 1281, Coquimbo, Chile
}

et al., 2008; Tukker \& Jansen, 2006). En segundo lugar y estrechamente asociada a la primera, está la preocupación acerca de las reservas mundiales de combustibles fósiles. En la actualidad estas reservas son limitadas y el incremento de su consumo (Owen et al., 2010) provoca importantes variaciones en sus precios y genera grandes conflictos en el control estratégico de estos recursos naturales.

Los EVs, como su nombre lo indica, son vehículos propulsados por motores eléctricos. Entre sus ventajas está la independencia de los combustibles tradicionales, la no emisión de gases a la atmosfera y una menor frecuencia entre mantenimientos (Delucchi, \& Lipman, (2001). Algunos países como Noruega, Francia, Reino Unido se han puesto como meta la sustitución total o parcial de los vehículos a combustión por autos eléctricos. Según el Observatorio Europeo de Combustible Alternativos, en el 2019 varios países de bloque mostraron importantes crecimientos en termino de venta de autos eléctricos, así como en el desarrollo de la infraestructura de carga. En general, el Parlamento Europeo estableció un objetivo para que el 40\% de los automóviles y furgonetas nuevos sean vehículos con cero o bajas emisiones para 2030 (European Parliament, 2018). Sin embargo, otras regiones no muestran el mismo avance y perspectiva en el tema. Por ejemplo, los países latinoamericanos muestran discretas iniciativas dirigidas al transporte público y un débil crecimiento en la venta de unidades a particulares. Este tema tiene mayor relevancia en esta área geográfica si se analiza el rápido crecimiento que ha tenido la venta de vehículos nuevos de tipo convencional, lo que incrementa emisión de gases (Rehermann \& Romero, 2018; González et al., 2019)

A pesar de las grandes ventajas la tecnología de los EVs aún tiene importantes desafíos como son la autonomía, el costo 
de producción y la infraestructura para la carga. Sin embargo, las baterías de litio (ion de litio y de estado sólido) parece ser una solución para los dos primeros. Su rendimiento es superior que el de las tecnologías tradicionales, en al menos un factor de 2.5, en términos de energía entregada (Dunn et al., 2011). Adicionalmente, proporciona alta potencia específica considerándose la batería idónea para alimentar la próxima generación de vehículos eléctricos, aunque su autonomía sigue siendo menor a la de los vehículos de combustión (Bruce et al., 2012; Choi, \& Aurbach, 2016; Schmuch et al., 2018). Estas baterías han revolucionado el almacenamiento de energía debido a su gran autonomía, rapidez de carga, menor peso, alta eficiencia y larga vida (Scrosati \& Garche, 2010).

Entre los principales componentes para la fabricación de estas baterías está el litio. Y en los países sudamericanos se concentran las mayores reservas de este mineral a nivel mundial, siendo Chile el principal exportador de este bien (U.S. Geological Survey, 2019). Sin embargo, para que estos países logren maximizar sus beneficios deben dejar de ser exportadores de commodities y convertirse en productores y exportadores de tecnologías en este tópico. Por otra parte, una apuesta hacia el desarrollo de los EVs en la región podría tener un impacto importante en la generación de empleo y la reducción de la contaminación (Sauer et al., 2015). En este sentido, dada la relación vehículo eléctrico- batería de litioreservas de litio disponibles en la región, la contribución de las investigaciones científicas sobre la temática resulta crucial para lograr estos objetivos. Así, este trabajo busca responder a la interrogante ¿Cuál ha sido la contribución de los investigadores latinoamericanos en la temática de los vehículos eléctricos? Aunque el foco de esta investigación no son las baterías de ion de litio, es conveniente analizar la participación que tiene las investigaciones este tema específico.

Las investigaciones sobre los vehículos eléctricos crecen a un ritmo que la evaluación de su progreso se vuelve obsoleto rápidamente (Scrosati \& Garche, 2010; Girishkumar et al., 2010). Para entender el comportamiento y las tendencias de un área de investigación específica, es frecuente el uso de la bibliometría que es una disciplina de la cienciometría que emplea métodos estadísticos y matemáticos (Moed et al.,1995; Archambault et al., 2009). Entre sus ventajas esta la disponibilidad de varios softwares que permiten el procesamiento y representación de la información de manera amigable (Chen, 2006; Van Eck \& Waltman, 2010; García et al., 2015). Su aplicación es transversal a cualquier tema en particular como por ejemplo la educación, los negocios, los combustibles, etc. (Martin et al., 2011; Chen et al., 2012; Liu et al., 2019). En particular, los estudios bibliométricos recientes relacionados a los vehículos eléctricos abordan temas como el impacto socioeconómico de la extracción de minerales de litio (Agusdinata et al., 2018), y la fiabilidad desde el punto de vista de la mantenibilidad de los vehículos eléctricos (Zhao et al., 2018). Otra línea de investigación dentro de los vehículos eléctricos, lo constituye las baterías de combustible de hidrogeno (Alvarez-Meaza et al., 2020).

En este contexto la presente investigación se enfoca en la región latinoamericana, y tiene como objetivo evaluar mediante un análisis bibliométrico la contribución científica de esta región en la temática de vehículos eléctricos. Para ello, se tuvieron en cuenta las investigaciones publicadas en revistas indexadas en la Web of Science (WOS) en el periodo 2001-2019. El estudio contempla el análisis de la evolución de la producción científica, la contribución por países, organizaciones, autores y revistas basada en la cantidad de publicaciones, números de citas y redes de coautoría. Incluye el análisis de palabras claves. También, se aplica el indicador de actividad científica para medir la especialización de los países en el tema, así como la relación entre productividad científica y financiamiento.

\section{Metodología}

Los datos fueron obtenidos de la Web of Science (WOS) en su colección principal. En la tabla 1 se detallan los aspectos relevantes de la búsqueda y resultados utilizando un procedimiento similar a (Alvarez-Meaza et al., 2019).

\footnotetext{
Tabla 1 Procedimiento de búsqueda de artículos científicos en la Web of Science sobre la temática de vehículos eléctricos y resultados obtenidos Fuente: Elaboración propia, 2020.
}

\begin{tabular}{lll}
\hline \multicolumn{1}{c}{ Aspectos } & \multicolumn{1}{c}{ Descripción } & Resultados \\
\hline & (TS = ((electric* NEAR/3 vehicle*) OR ("hybrid & \\
Ecuación & electric" NEAR/3 vehicle*) OR (electric* & \\
& NEAR/3 car) OR ("hybrid electric" NEAR/3 car) & \multirow{2}{*}{ 18748 docs } \\
& OR (electric* NEAR/3 cars) OR ("hybrid electric" & \\
& NEAR/3 cars))) & \\
Filtro países & (Costa Rica O Colombia O México O Chile O & \multirow{2}{*}{360 docs } \\
& Argentina O Cuba O Brazil O Panamá O Trinid & \\
Periodo & Tobago O Uruguay O Ecuador) & \\
Tipo de documentos & 2001-2019 & \\
Fecha de la búsqueda & Artículos y contribuciones a congresos & \\
\hline
\end{tabular}


El procesamiento de los datos se realizó mediante el software gratuito VOSviewer que permite construir y visualizar redes bibliométricas de países, organizaciones, autores, revistas, y palabras claves (Van Eck y Waltman, 2007). Entre las ventajas de su uso están la capacidad de procesamiento de gran cantidad de datos y la disponibilidad de literatura con descripción metodológica de su manejo (Van Eck, et al., 2006; Van Eck y Waltman, 2007; Van Eck y Waltman, 2010).

El procesamiento y análisis de los datos se realizó siguiendo la estructura que se muestra en la figura 1 .
Figura 1 Estructura del análisis bibliométrico.

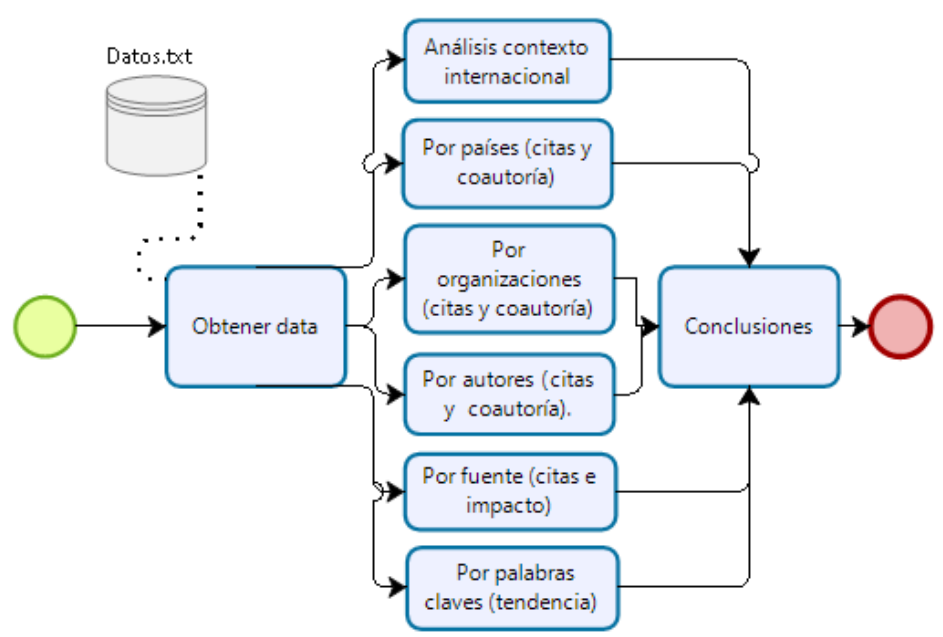

P: Producción total de documentos en el mundo para todas las temáticas.

Debido a la gran cantidad de documentos existentes, se recomienda que el IA se calcule año por año y se analice su evolución en el tiempo. Cuando el IA es mayor a 100 se considera que el país tiene especialización en el tema.

Por último, se calculó el coeficiente de correlación de Pearson para identificar la relación existente entre la productividad científica en la temática de los EVs en cada país y la cantidad de publicaciones sobre EVs financiadas por las principales agencias de financiamiento científico estatal de cada país analizado.

\section{Resultados y Análisis}

\subsection{Análisis de la producción científica latinoamericana relacionada a vehículos eléctricos en el contexto internacional}

La participación promedio de la literatura Latinoamérica en el conocimiento sobre vehículos eléctricos en el contexto mundial es baja, solo un 1.8\%. Es relevante que presenta una tendencia creciente exponencial similar a los resultados de los investigadores del mundo para la temática (Figura 2). $\mathrm{P}_{\mathrm{k}}$ : Producción
para la temática $\mathrm{k}$. 
Figura 2 Publicaciones anuales sobre la temática de los vehículos eléctricos de los países latinoamericanos vs el mundo en periodo 2001-2019.

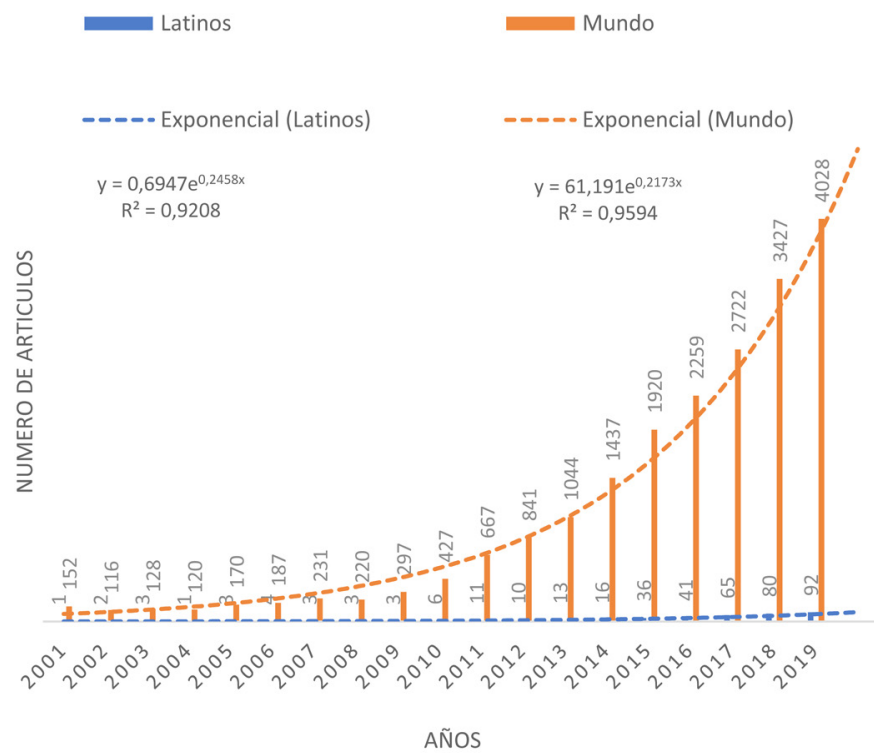

En la región, la investigación sobre esta temática está liderada por Brasil con 170 documentos citados 3310 veces, seguido por México (59 con 629 citas) y Colombia (56 con 346 citas). En el cuarto lugar aparece Chile con 50 documentos que, sin embargo, cuenta con un mayor número de citas (1539) que los dos países que lo preceden. Los países donde existe un mayor flujo de conocimiento con Latinoamérica, reflejado a través de las citas de documentos son Estados Unidos (USA), España, Italia e Inglaterra, entre otros (Figura 3).
Figura 3 Mapa de citación de documentos generados por países latinoamericanos en estudios sobre vehículos eléctricos en el período 2001- 2019.

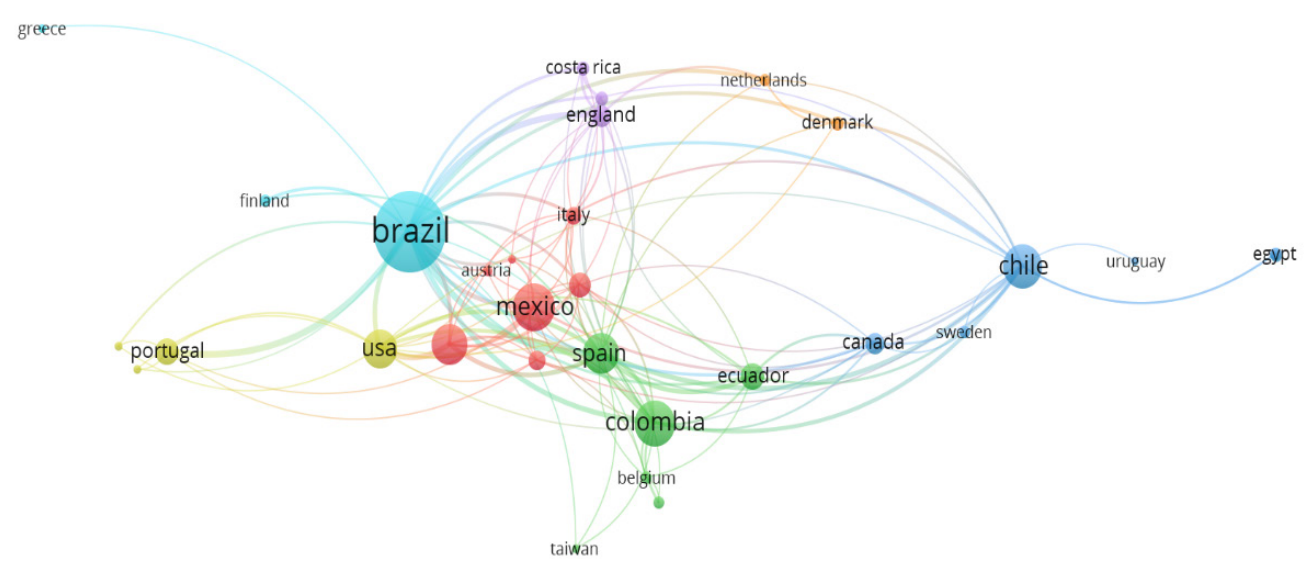

En general, los países latinoamericanos muestran baja especialización en la temática de los vehículos eléctricos. El índice de actividad científica (IA) posiciona a Colombia como el país más especializado con un valor promedio de 91 , seguido por Chile con 49 y México con 41 (Figura 4). Chile es el único país con tendencia creciente. 
Figura 4 Índice de actividad científica (IA) para los países latinoamericanos en publicaciones sobre vehículos eléctricos en el período 2015-2019.

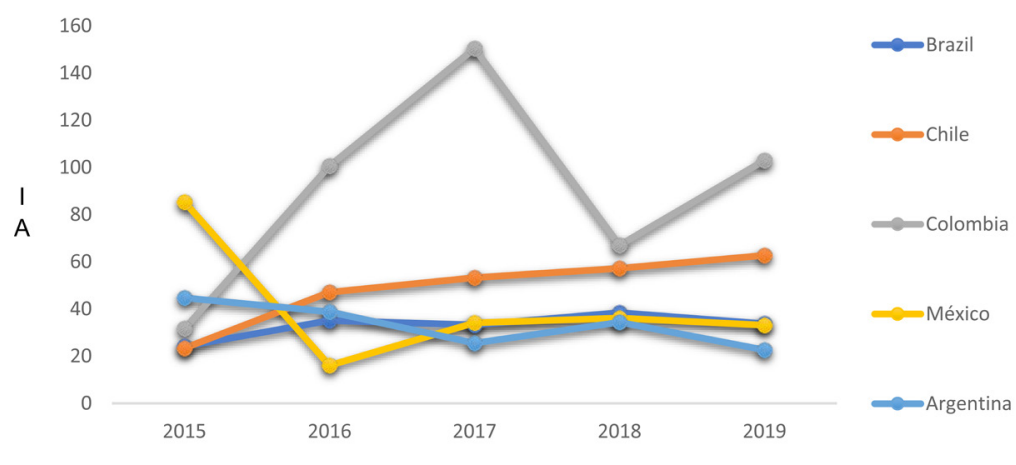

La colaboración de autoría entre países latinoamericanos es baja comparada con países de otras regiones, con clústeres de un máximo de tres países (Figura 5). Brasil lidera con 30 links y una fuerza total de asociación de 114. Le siguen Chile con 17 y Colombia con 16 conexiones, ambos con similar número de colaboraciones. Sin embargo, Chile colabora con mayor frecuencia según la fuerza total de asociación 42 vs
32. Respecto a la colaboración con países de otras regiones destacan las redes con Estados Unidos, España, China, Francia, entre otros. Estas redes son muy importe para los países latinoamericanos porque en muchos casos le permite acceder a recursos económicos y la transferencia de nuevos conocimientos (Jung, N. \& Ruiz-León, A., 2018).
Figura 5 Mapa de coautoría de los países latinoamericanos en estudios sobre vehículos eléctricos en el período 2001- 2019.

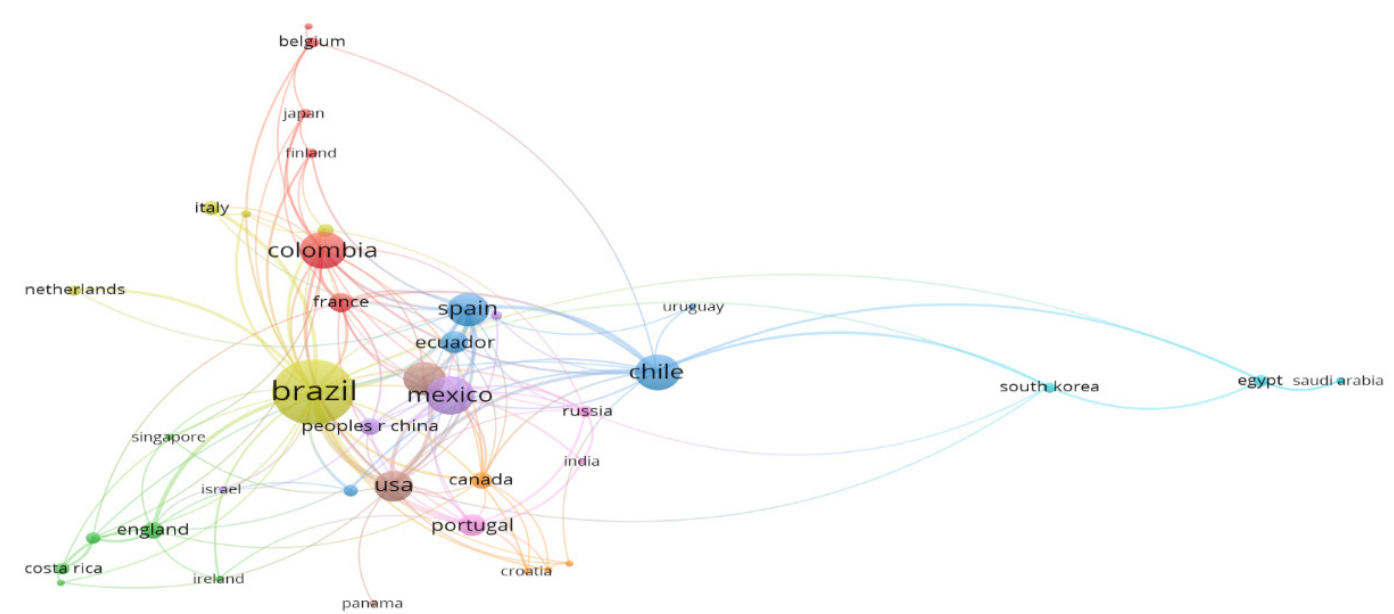

\subsection{Análisis de producción, citas y coautoría por organizaciones}

El top 10 de las organizaciones latinoamericanas está liderado por la Universidad de São Paulo (Brasil) con 19 publicaciones (Tabla 2). Por otra parte, la Pontificia Universidad Católica de Chile (Chile) destaca en cantidad de citas por documentos (CPP) con 84, muy superior al resto de las otras organizaciones. Como se puede apreciar el promedio de citas que alcanzan estos documentos es bajo. Esto puede estar influenciado porque es una temática emergente en la región y sus primeras publicaciones fueron a principio del siglo XXI con muy baja productividad de documentos en los primeros 10 años de análisis. 
Tabla 2 Top 10

de organizaciones

latinoamericanas en

el conocimiento sobre

vehículos eléctricos en el

período 2001-2019.

Fuente: Elaboración propia a

partir de WOS, 2020

\begin{tabular}{lcccc}
\hline \multicolumn{1}{c}{ Organizaciones } & Países & Récords & \% & CPP \\
\hline Universidad de São Paulo & Brasil & 19 & 4.8 & 12 \\
Universidad Nacional de Colombia & Colombia & 17 & 4.3 & 4 \\
Universidad Estatal de Campinas & Brasil & 15 & 3.8 & 19 \\
Universidad Federal de Río de Janeiro & Brasil & 15 & 3.8 & 33 \\
Universidad Nacional de Río Cuarto & Argentina & 15 & 3.8 & 32 \\
Pontificia Universidad Católica de Chile & Chile & 13 & 3.3 & 84 \\
Universidad Federal de Santa Catarina & Brasil & 12 & 3.0 & 14 \\
Universidad Nacional Autónoma de México & México & 11 & 2.7 & 18 \\
Universidad de Chile & Chile & 10 & 2.5 & 16 \\
Universidad Técnica Federico Santa María & Chile & 10 & 2.5 & 10 \\
\hline
\end{tabular}

Siguiendo la tendencia de baja colaboración entre países de Latinoamérica, se encuentran las organizaciones de esta región, con las menos colaborativas situadas en la zona periférica (Figura 6). En el centro del mapa se ubican las instituciones con más relaciones de coautoría como la Universidad de Sao Paulo (Brasil), y la Universidad Nacional de Colombia (Colombia). Como se puede apreciar existe un gran desafío en cuanto a aumentar la colaboración en número y frecuencia entre las organizaciones de la región. Por otra parte, es necesario que las instituciones latinoamericanas fortalezcan sus intercambios las con las organizaciones líderes en la temática, con el fin de lograr una transferencia acelerada de los conocimientos.
Figura 6 Mapa de coautoría de organizaciones en estudios sobre vehículos 2019. eléctricos el periodo 2001-

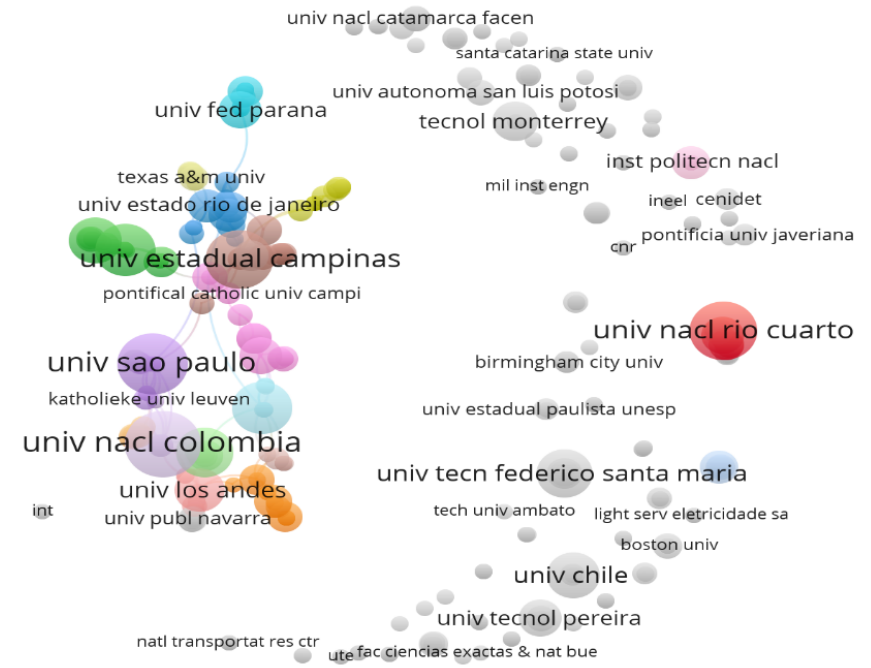

\subsection{Análisis de producción y coautoría por autores}

De un total 1325 autores vinculados a la investigación sobre autos eléctricos en Latinoamérica, solo 50 presentan tres o más documentos en coautoría. En rojo se presenta el clúster donde destaca Marcos J. Rider de la Universidad Estatal de
Campinas (Brasil), en el verde Fernanda C. Correa de esta misma universidad, y en el azul Cristian H De Angelo de la Universidad Nacional Rio Cuarto (Argentina). Estos grupos se constituyen al interior de estas universidades (Figura 7). Esto refuerza la idea que existe una gran necesidad de crear redes de colaboración en la temática para lograr una mayor integración y aceleración de la producción científica. 
Figura 7 Mapa de coautoría de autores latinoamericanos en estudios sobre vehículos electicos (2001-2019).

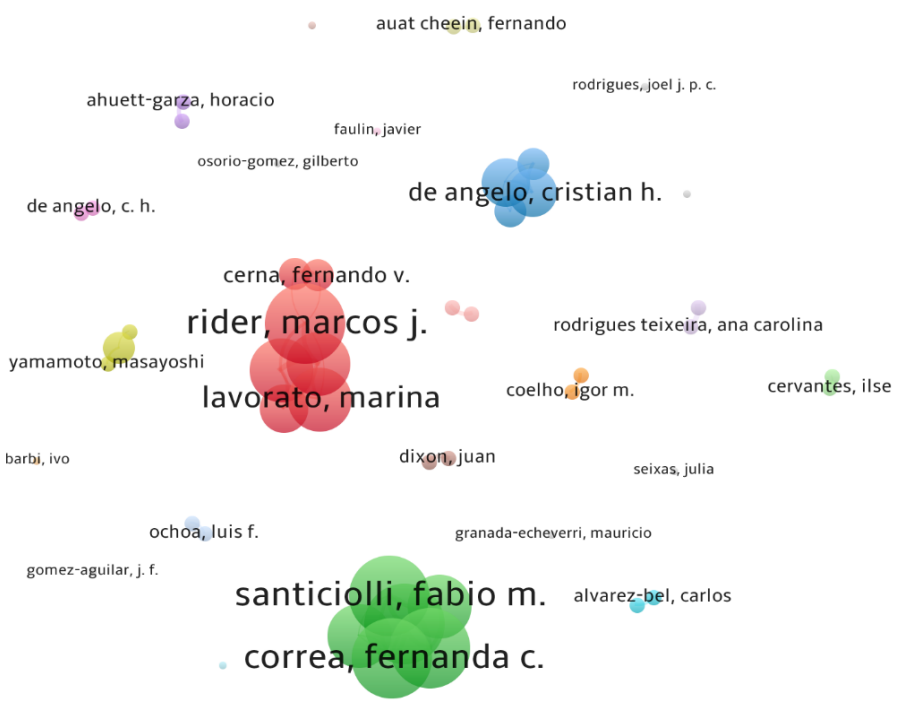

Entre los autores latinoamericanos con mayor relevancia en la temática se encuentran Jorge Moreno, Micah E. Ortúzar y Juan W. Dixon con su artículo "Energy-management system for a hybrid electric vehicle, using ultracapacitors and neural networks" que alcanza 416 citas, y otro artículo sobre la implementación y evaluación de este tema con 279 citas. La investigación de Mattos y Noranhan aparece con 403 citas a pesar de contar con fecha más reciente de publicación (Tabla
3). La relevancia de estos trabajos atendiendo al indicador número de citas, es menor a las investigaciones más destacadas temática a nivel mundial que superan las 5000 citas. Estos trabajos que alcanzan mayor número de citas a nivel global son estudios relacionados las baterías de ion de litio, sin embargo, las investigaciones latinoamericanas más relevante apuntan hacías otros aspectos de los EVs,
Tabla 3 Autores citados en investigaciones sobre vehículos eléctricos (2001-2019)

Fuente: Elaboración propia a partir de WOS, 2020 latinoamericanos más

\begin{tabular}{|c|c|c|c|c|}
\hline Autores & Año & País & Organización & $\mathbf{N}^{0}$ Citas \\
\hline $\begin{array}{l}\text { Moreno, J., Ortuzar, M. E., \& } \\
\text { Dixon }\end{array}$ & 2006 & Chile & $\begin{array}{c}\text { Pontificia Universidad } \\
\text { Católica de Chile }\end{array}$ & 416 \\
\hline Mattos, L. V., \& Noronha, F. B & 2012 & Brasil & $\begin{array}{l}\text { Fluminense Fed Univ - } \\
\text { Natl Inst Technol INT }\end{array}$ & 403 \\
\hline $\begin{array}{l}\text { Ortuzar, M., Moreno, J., \& } \\
\text { Dixon }\end{array}$ & 2007 & Chile & $\begin{array}{l}\text { Pontificia Universidad } \\
\text { Católica de Chile }\end{array}$ & 279 \\
\hline Pomilio, J. A & 2005 & Brasil & $\begin{array}{c}\text { Universidad Estatal de } \\
\text { Campinas }\end{array}$ & 235 \\
\hline Gomez, J. C. & 2003 & Argentina & Univ Nacl Rio Cuarto & 185 \\
\hline
\end{tabular}

\subsection{Análisis de palabras claves}

Entre las 2232 palabras claves utilizadas en los estudios analizados, solamente 87 tienen, al menos, cinco frecuencias de uso (Figura 8). Destacan entre las más relevantes "Vehículos eléctricos" y "Vehículo eléctrico", "Optimización", "Sistema", "Batería", "Híbrido", "Diseño", "Energía" y "Administración". Llama la atención que "Baterías de ion de litio" no se encuentre entre las palabras con mayor frecuencia. 
Figura 8 Mapa de coocurrencia de palabras claves en estudios sobre vehículos eléctricos de autores latinoamericanos (2001- 2019).

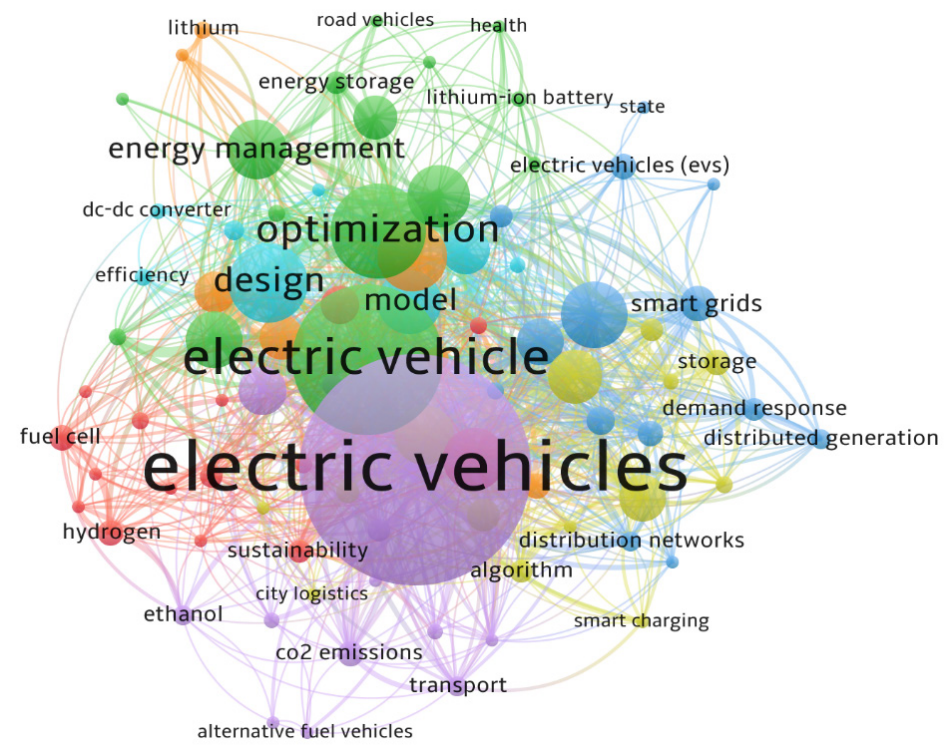

IEEE Transactions On Industrial Electronics alcanza mayor cantidad de citas por documento (88). Cabe destacar que aquellas revistas de menor factor de impacto presentan menos citaciones por documento. Como se puede apreciar no hay ninguna revista latinoamericana indexada en WOS que tenga contribución destacada en la publicación de artículos relacionados con los EVs. Esta situación puede estar relacionada con la poca cantidad de revistas latinoamericanas que están indexada en JCR, además del bajo FI de estas (Crespo-Gascón, et al., 2019).
Tabla 4 Top 10 de revistas

con artículos sobre

vehículos eléctricos de

autores latinoamericanos

(2001-2019).

Fuente: Elaboración propia a

partir de WOS, 2020

\begin{tabular}{|c|c|c|c|c|c|c|}
\hline Revista & País & Récord & $\%$ & CPP & FI & $\mathbf{Q}$ \\
\hline $\begin{array}{l}\text { Energies } \\
\text { IEEE Transactions on }\end{array}$ & Switzerland & 18 & 4.6 & 5 & 2.707 & Q3 \\
\hline $\begin{array}{l}\text { Industrial Electronics } \\
\text { IEEE Latin America }\end{array}$ & EE. UU. & 15 & 3.8 & 88 & 7.503 & Q1 \\
\hline $\begin{array}{l}\text { Transactions } \\
\text { IEEE Transactions on }\end{array}$ & EE. UU. & 14 & 3.6 & 2 & 0.804 & Q4 \\
\hline Vehicular Technology & EE. UU. & 12 & 3.1 & 20 & 5.339 & Q1 \\
\hline $\begin{array}{l}\text { Applied Energy } \\
\text { Energy } \\
\text { Renewable Sustainable }\end{array}$ & $\begin{array}{l}\text { England } \\
\text { England }\end{array}$ & $\begin{array}{l}11 \\
10\end{array}$ & $\begin{array}{l}2.8 \\
2.5\end{array}$ & $\begin{array}{l}29 \\
19\end{array}$ & $\begin{array}{l}8.426 \\
5.537\end{array}$ & $\begin{array}{l}\text { Q1 } \\
\text { Q1 }\end{array}$ \\
\hline Energy Reviews & England & 10 & 2.5 & 33 & 10.556 & Q1 \\
\hline $\begin{array}{l}\text { Journal Of Power Sources } \\
\text { Electric Power Systems }\end{array}$ & Netherlands & 9 & 2.3 & 24 & 7.467 & Q1 \\
\hline $\begin{array}{l}\text { Research } \\
\text { Journal Of Cleaner }\end{array}$ & Switzerland & 8 & 2.0 & 12 & 3.022 & Q2 \\
\hline Production & England & 8 & 2.0 & 12 & 6.395 & Q1 \\
\hline
\end{tabular}




\subsection{Análisis de financiamiento}

En la tabla 5 se muestra el número de investigaciones que fueron financiadas por las principales agencias de financiamiento de cada país latinoamericano, para la temática de los EVs. Brasil cuenta con el mayor número de agencias y esto se ve reflejado en el número de publicaciones que logró este país. En segundo lugar, aparecen agencias de México y Chile, sin embargo, México logró mayor número de publicaciones en el tema. El caso de Colombia su agencia es la que menor porcentaje de proyectos financia, pero es el tercer país con mayor número de publicaciones.
El enfoque de la actividad científica puede estar definida por políticas internas de las organizaciones o por un marco país, donde se definen y potencian las áreas de conocimientos de interés nacional, las cuales se financian mediante instrumentos de fomento. En el caso de la temática de los EVs para Latinoamérica se aprecia que existe una correlación positiva de 0,95 entre financiamiento gubernamental y cantidad de publicaciones. En general se identificó que el $52 \%$ de las investigaciones son financiadas estas agencias. Otro grupo importante de investigaciones son financiadas o cofinanciadas por organizaciones internacionales.
Tabla 5 Principales

agencias de financiamiento

para investigaciones sobre

Fuente: Elaboración propia a

partir de WOS, 2020
EVs (2001-2019).

\begin{tabular}{llcc}
\hline Agencia estatal de financiamiento & País & $\begin{array}{c}\text { Doc. } \\
\text { por } \\
\text { País }\end{array}$ & $\begin{array}{c}\text { Doc. } \\
\text { Financiado }\end{array}$ \\
\hline NATIONAL COUNCIL CNPQ & Brasil & & 59 \\
CAPES & Brasil & 170 & 42 \\
FUNDACAO DE AMPARO A PESQUISA (FAPESP) & Brasil & & 23 \\
MINAS GERAIS STATE RESEARCH FOUNDATION FAPEMIG & Brasil & & 12 \\
CONACYT & México & 59 & 20 \\
CONICYT & Chile & 50 & 20 \\
CONICET & Argentina & 43 & 13 \\
ANPCYT & Argentina & & 8 \\
COLCIENCIAS & Colombia & 56 & 7 \\
SECRETARÍA DE TECNOLOGÍA E INNOVACIÓN & Ecuador & 19 & 1 \\
NO INFORMA (AGENCIA) & Costa Rica & 6 & 0 \\
NO INFORMA (AGENCIA) & Cuba & 2 & 0 \\
NO INFORMA (AGENCIA) & Panamá & 2 & 0 \\
NO INFORMA (AGENCIA) & Uruguay & 2 & 0 \\
NO INFORMA (AGENCIA) & Trinidady & 2 & 0 \\
\hline
\end{tabular}

\section{Conclusiones}

El panorama científico en la temática de vehículos eléctricos en Latinoamérica esta matizado por una baja productividad de sus organizaciones y autores. Para mejorar su productividad se debe mejorar la colaboración con otros países que concentran las instituciones líderes en la temática. A su vez es necesario fortalecer la colaboración entre instituciones y autores de la región.

El crecimiento de las investigaciones sobre los EVs tiene un crecimiento exponencial en la región. Sin embargo, no es suficiente para lograr un mayor protagonismo en consonancia con el estándar mundial, y que les permita una rápida evolución hacia implantación de esta tecnología.

El IA demuestra que aquellos países con mayor potencial científico como el caso de Brasil y México tienen poco enfoque hacia la temática. En el caso de Brasil tiene una participación destacada la Universidad de Sao Paulo en cuanto a cantidad de publicaciones y coautoría, pero bajo en comparación con las instituciones líderes en la temática a nivel mundial.
Los autores más destacados publican en las principales revistas especializadas en la temática como son Renewable Sustainable Energy Reviews, Applied Energy, y Energy, todas con buen factor de impacto, lo que evidencia que existe buena calidad en las investigaciones realizadas.

El análisis de palabras claves evidencia poco enfoque hacia las investigaciones relacionadas con desarrollo de las baterías de litio. Atendiendo al potencial productivo que tienen los autos eléctricos y las grandes reservas de litio existentes en la región. Es importante que las principales agencias de financiamiento científico como son, el Consejo Nacional De Desarrollo Científico Y Tecnológico (CNPQ), la Fundacao De Amparo A Pesquisa Do Estado De Sao Paulo (FAPESP) la Comisión Nacional De Investigación Científica Y Tecnológica (CONICYT) el Consejo Nacional De Ciencia Y Tecnología (CONACYT) el Consejo Nacional De Investigaciones Científicas Y Técnicas (CONICET), entre otras, implementen una política con enfoque hacia los temas relacionados con los vehículos eléctricos y al uso de tecnología asociadas al litio. 


\section{Referencias bibliográficas}

Alvarez-Meaza I., Zarrabeitia-Bilbao E., Rio-Belver R.M., \& de Alegría I.M. (2019) Mapping Scientific and Technological Patterns: Hybrid Vehicles. In: Reis J., Pinelas S., Melão N. (eds) Industrial Engineering and Operations Management I. IJCIEOM 2018. Springer Proceedings in Mathematics \& Statistics, vol 280. Springer, Cham. doi.org/10.1007/978-3-030-149697_13

Alvarez-Meaza, I., Zarrabeitia-Bilbao, E., Rio-Belver, R. M., \& Garechana-Anacabe, G. (2020). FuelCell Electric Vehicles: Plotting a Scientific and Technological Knowledge Map. Sustainability, 12(6), 2334. doi:10.3390/su12062334

Archambault, É., Campbell, D., Gingras, Y., \& Larivière, V. (2009). Comparing bibliometric statistics obtained from the Web of Science and Scopus. Journal of the American Society for Information Science and Technology, 60(7), 1320-1326. doi:10.1002/asi.21062

Agusdinata, D. B., Liu, W., Eakin, H., \& Romero, H. (2018). Socio-environmental impacts of lithium mineral extraction: towards a research agenda. Environmental Research Letters. doi:10.1088/1748-9326/aae9b1

Bruce, P. G., Freunberger, S. A., Hardwick, L. J., \& Tarascon, J.-M. (2012). Li-O2 and Li-S batteries with high energy storage. Nature Materials, 11(1), 19-29. doi:10.1038/ nmat3191

Chen, C. (2006). CiteSpace II: Detecting and visualizing emerging trends and transient patterns in scientific literature. Journal of the American Society for Information Science and Technology, 57(3), 359-377. doi:10.1002/asi.20317

Chen, H., Chiang, R., \& Storey V. (2012). Business Intelligence and Analytics: From Big Data to Big Impact. MIS Quarterly, 36(4), 1165. doi:10.2307/41703503

Choi, J. W., \& Aurbach, D. (2016). Promise and reality of post-lithium-ion batteries with high energy densities. Nature Reviews Materials, 1(4). doi:10.1038/ natrevmats. 2016.13

Crespo-Gascón, S., Tortosa, F. S., \& Guerrero-Casado, J. (2019). Producción de revistas científicas en América Latina y El Caribe en Scopus, Journal Citation Reports y Latindex en el área de los recursos naturales: su relación con variables económicas, ambientales, y de inversión en investigación. Revista Española de Documentación Científica, 42 (1), 224.
Delucchi, M. A., \& Lipman, T. E. (2001). An analysis of the retail and lifecycle cost of battery-powered electric vehicles. Transportation Research Part D: Transport and Environment, 6(6), 371-404. doi:10.1016/s13619209(00)00031-6

Dunn, B., Kamath, H., \& Tarascon, J.-M. (2011). Electrical Energy Storage for the Grid: A Battery of Choices. Science, 334(6058), 928 935. doi:10.1126/ science. 1212741

European Parliament (2018). More Electric Cars on EU Roads by $2030 \mid$ Nieuws|Europees Parlement.

Available online: http://www.europarl.europa.eu/news/nl/ press-room/20180911IPR13114/more-electric-cars-oneuroads-by-2030 (accessed on 21 December 2020)

Fargione, J., Hill, J., Tilman, D., Polasky, S., \& Hawthorne, P. (2008). Land clearing and the biofuel carbon debt. Science, 319(5867), 1235-1238.DOI: 10.1126/ science. 1152747

García, A., Pardo, A., Ferrer, A., Peset, M. F., \& González, L. (2015). Herramientas de análisis de datos bibliográficos y construcción de mapas de conocimiento: Bibexcel y Pajek. BiD. Textos Universitaris de Biblioteconomia i Documentació, (34), 1-8. dx.doi.org/10.1344/ BiD2015.34.22

Girishkumar, G., McCloskey, B., Luntz, A. C., Swanson, S., \& Wilcke, W. (2010). Lithium-Air Battery: Promise and Challenges. The Journal of Physical Chemistry Letters, 1(14), 2193-2203. doi:10.1021/jz1005384

Goodenough, J. B., \& Kim, Y. (2010). Challenges for Rechargeable Li Batteries $\uparrow$. Chemistry of Materials, 22(3), 587-603. doi:10.1021/cm901452z

González, L. G., Siavichay, E., \& Espinoza, J. L. (2019). Impact of $\mathrm{EV}$ fast charging stations on the power distribution network of a Latin American intermediate city. Renewable and Sustainable Energy Reviews, 107, 309-318. doi:10.1016/j.rser.2019.03.017

Gomez, J. C., \& Morcos, M. M. (2003). Impact of EV battery chargers on the power quality of distribution systems. IEEE Transactions on Power Delivery, 18(3), 975-981. doi:10.1109/tpwrd.2003.813873

Hu, Y., Sun, J., Li, W., \& Pan, Y. (2013). A scientometric study of global electric vehicle research. Scientometrics, 98(2), 1269-1282. doi:10.1007/s11192-013-1067-8

Liu, H., Hong, R., Xiang, C., Lv, C. y Li, H. (2019). Visualization and analysis of mapping knowledge domains for spontaneous combustion studies. Fuel, 116598. doi: 10.1016 / j.fuel.2019.116598 
Lu, L., Han, X., Li, J., Hua, J., \& Ouyang, M. (2013). A review on the key issues for lithium-ion battery management in electric vehicles. Journal of Power Sources, 226, 272288. doi:10.1016/j.jpowsour.2012.10.06

Jung, N., \& Ruiz-León, A. A. (2018). Lo local y lo global de la colaboración científica: ¿qué significa, y cómo visualizarlo y medirlo? Revista Española de Documentación Científica, 41 (2): e203. doi. org/10.3989/redc.2018.2.1463

Ortuzar, M., Moreno, J., \& Dixon, J. (2007). UltracapacitorBased Auxiliary Energy System for an Electric Vehicle: Implementation and Evaluation. IEEE Transactions on Industrial Electronics, 54(4), 2147-2156. doi:10.1109/ tie. 2007.894713

Owen, N. A., Inderwildi, O. R., \& King, D. A. (2010). The status of conventional world oil reserves-Hype or cause for concern? Energy Policy, 38(8), 4743-4749. doi:10.1016/j.enpol.2010.02.026

Mattos, L. V., Jacobs, G., Davis, B. H., \& Noronha, F. B. (2012). Production of Hydrogen from Ethanol: Review of Reaction Mechanism and Catalyst Deactivation. Chemical Reviews, 112(7), 4094-4123. doi:10.1021/ cr2000114

Martin, S., Diaz, G., Sancristobal, E., Gil, R., Castro, M., \& Peire, J. (2011). New technology trends in education: Seven years of forecasts and convergence. Computers \& Education, 57(3), 1893-1906. doi:10.1016/j. compedu.2011.04.003

Moed, H. F. (2009). New developments in the use of citation analysis in research evaluation. Archivum Immunologiae et Therapiae Experimentalis, 57(1), 13-18. doi:10.1007/ s00005-009-0001-5

Moed, H. F., De Bruin, R. E., \& Van Leeuwen, T. N. (1995). New bibliometric tools for the assessment of national research performance: Database description, overview of indicators and first applications. Scientometrics, 33(3), 381-422. doi:10.1007/bf02017338

Moreno, J., Ortuzar, M. E., \& Dixon, J. W. (2006). Energymanagement system for a hybrid electric vehicle, using ultracapacitors and neural networks. IEEE Transactions on Industrial Electronics, 53(2), 614-623. doi:10.1109/ tie. 2006.870880

Rehermann, F., \& Pablo-Romero, M. (2018). Economic growth and transport energy consumption in the Latin American and Caribbean countries. Energy Policy, 122, 518-527. doi:10.1016/j.enpol.2018.08.006
Sauer, I. L., Escobar, J. F., da Silva, M. F. P., Meza, C. G., Centurion, C., \& Goldemberg, J. (2015). Bolivia and Paraguay: A beacon for sustainable electric mobility? Renewable and Sustainable Energy Reviews, 51, 910925. doi:10.1016/j.rser.2015.06.038

Schubert, A., Glänzel, W., \& Braun, T. (1989). Scientometric datafiles. A comprehensive set of indicators on 2649 journals and 96 countries in all major science fields and subfields 1981-1985. Scientometrics, 16(1-6), 3-478. doi:10.1007/bf02093234

Schmuch, R., Wagner, R., Hörpel, G., Placke, T., \& Winter, M. (2018). Performance and cost of materials for lithium-based rechargeable automotive batteries. Nature Energy, 3(4), 267-278. doi:10.1038/s41560-018-0107-2

Scrosati, B., \& Garche, J. (2010). Lithium batteries: Status, prospects and future. Journal of Power Sources, 195(9), 2419-2430. doi:10.1016/j.jpowsour.2009.11.048

Solero, L., Lidozzi, A., \& Pomilio, J. A. (2005). Design of Multiple-Input Power Converter for Hybrid Vehicles. IEEE Transactions on Power Electronics, 20(5), 10071016. doi:10.1109/tpel.2005.854020

Van Eck N. J., \& Waltman L. (2010). Software survey: VOSviewer, a computer program for bibliometric mapping. Scientometrics; 84(2):523-38.

Van Eck, N. J., \& Waltman, L. (2007). Bibliometric mapping of the computational intelligence field. International Journal of Uncertainty, Fuzziness and KnowledgeBased Systems, 15(5), 625-645.

Van Eck, N. J., Waltman, L., Van den Berg, J., \& Kaymak, U. (2006). Visualizing the computational intelligence field. IEEE Computational Intelligence Magazine, 1(4), 6-10.

Twigg, M. V. (2007). Progress and future challenges in controlling automotive exhaust gas emissions. Applied Catalysis B: Environmental, 70(1-4), 2-15. doi:10.1016/j.apcatb.2006.02.029

Tukker, A., \& Jansen, B. (2006). Environmental Impacts of Products: A Detailed Review of Studies. Journal of Industrial Ecology, 10(3), 159-182. doi:10.1162/ jiec.2006.10.3.159

Thomas, C. D., Cameron, A., Green, R. E., Bakkenes, M., Beaumont, L. J., Collingham, Y. C., ... Williams, S. E. (2004). Extinction risk from climate change. Nature, 427(6970), 145-148. doi:10.1038/nature02121 
U.S. Geological Survey, (2019), Mineral commodity summaries 2019: U.S. Geological Survey, 200 p. doi. org/10.3133/70202434.

Walther, G.-R., Post, E., Convey, P., Menzel, A., Parmesan, C., Beebee, T. J. C., ... Bairlein, F. (2002). Ecological responses to recent climate change. Nature, 416(6879), 389-395. doi:10.1038/416389a
Zhao, X., Wang, S., \& Wang, X. (2018). Characteristics and trends of research on new energy vehicle reliability based on theweb of science. Sustainability (Switzerland), 10(10), 1-25. doi.org/10.3390/su10103560 